\title{
Evaluation of Guanfacine as a Potential Medication for Alcohol Use Disorder in Long-Term Drinking Rats: Behavioral and Electrophysiological Findings
}

\author{
Ida Fredriksson ',3, Nitya Jayaram-Lindström ${ }^{1,3}$, Malin Wirf', Erik Nylander', Erica Nyström', \\ Kent Jardemark ${ }^{2}$ and Pia Steensland*, I \\ 'Department of Clinical Neuroscience, Karolinska Institutet, Stockholm, Sweden; ${ }^{2}$ Department of Physiology and Pharmacology, Karolinska \\ Institutet, Stockholm, Sweden
}

\begin{abstract}
One of the main treatment challenges in alcohol use disorder (AUD) is the high rate of craving in combination with decreased cognitive functioning including impaired decision making and impulse control that often lead to relapse. Recent studies show that guanfacine, an $\alpha$-2-adrenoceptor agonist and FDA-approved ADHD medication, attenuates stress-induced relapse of several drugs of abuse including alcohol. Here we evaluated guanfacine's effects on voluntary alcohol intake, the alcohol deprivation effect (ADE), alcohol seeking behavior, and cue/priming-induced reinstatement in Wistar rats that had voluntarily consumed alcohol for at least 2 months before treatment. In addition, guanfacine's ability to regulate glutamatergic neurotransmission was evaluated through electrophysiological recordings in medial prefrontal cortex (mPFC) slices prepared from long-term drinking rats (and alcohol-naive controls) that had received three daily guanfacine $(0.6 \mathrm{mg} / \mathrm{kg} / \mathrm{day})$ or vehicle injections in vivo. Guanfacine decreased alcohol intake in high, but not low, alcohol-consuming rats and the effects were generally more long lasting than that of the AUD medication naltrexone. Repeated guanfacine treatment induced a long-lasting decrease in alcohol intake, persistent up to five drinking sessions after the last injection. In addition, guanfacine attenuated the ADE as well as alcohol seeking and cue/priming-induced reinstatement of alcohol seeking. Finally, subchronic guanfacine treatment normalized an alcohol-induced dysregulated glutamatergic neurotransmission in the mPFC. These results support previous studies showing that guanfacine has the ability to improve prefrontal connectivity through modulation of the glutamatergic system. Together with the fact that guanfacine appears to be clinically safe, these results merit evaluation of guanfacine's clinical efficacy in AUD individuals.

Neuropsychopharmacology (20 I5) 40, I I30-1 |40; doi: I0.1038/npp.20 I4.294; published online 26 November 20 I4
\end{abstract}

\section{INTRODUCTION}

Alcohol use disorder (AUD) is a chronic relapsing disorder significantly contributing to the global burden of disease (Rehm et al, 2009). Attenuation of alcohol's reinforcing properties and prevention of relapse has historically been the major focus in medication development. Recently, the focus has shifted into understanding the role of neurobiological dysregulations in the prefrontal cortex (PFC) induced by chronic alcohol and drug use (eg, a heightened stress system and cognitive impairments) (Goldstein and Volkow, 2011) and evaluating these as potential treatment targets for AUD (Goddard et al, 2010; Gamo and Arnsten, 2011; Holmes et al, 2013). In particular, the noradrenergic and glutamatergic systems in the PFC have been identified

*Correspondence: Dr P Steensland, Department of Clinical Neuroscience, Karolinska Institutet, Solna, R5:01, Stockholm SE-17I76, Sweden, Tel: +46 8 517748888, Fax: +46 8 12349602,

E-mail: pia.steensland@ki.se

${ }^{3}$ These authors contributed equally to this work.

Received 18 June 2014; revised 18 October 2014; accepted 23 October 2014; accepted article preview online 31 October 2014 as potential core substrates regulating the involved deficits as they might mediate, for example, compulsive drug use and/or cue- and stress-induced craving that in turn may trigger relapse (Goddard et al, 2010; Gamo and Arnsten, 2011; Holmes et al, 2013).

Guanfacine, an $\alpha$-2-adrenoreceptor agonist, was recently FDA approved for the treatment of attention-deficit hyperactivity disorder (ADHD) in children and adolescents (Sallee et al, 2009a,b; Sallee and Eaton, 2010). The potential therapeutic efficacy of guanfacine in ADHD was based on the extensive preclinical work done by Arnsten and colleagues (Arnsten, 2010; Arnsten and Jin, 2014) showing that guanfacine has the ability to improve prefrontal connectivity, possibly through modulation of the glutamatergic system including N-Methyl-D-aspartate (NMDA) and $\alpha$-Amino-3-hydroxy-5-methyl-4-isoxazolepropionic acid (AMPA) receptors. Guanfacine's ability to also improve working memory deficits produced by the NMDA receptor antagonist phencyclidine (Marrs et al, 2005) further highlights the interconnection between the noradrenergic and glutamatergic systems in disorders characterized by cognitive deficits such as ADHD, but also possibly in dependence 
disorders. Therefore, evaluation of guanfacine's potential as a novel medication for dependence disorders has recently been initiated. Indeed, in a human brain imaging study, guanfacine attenuated cocaine craving, arousal, and anxiety while increasing PFC activity in cocaine-dependent individuals (Fox et al, 2012). Guanfacine also attenuated cocaine and alcohol craving in cocaine-dependent individuals co-abusing alcohol (Fox et al, 2014). Furthermore, guanfacine blunted alcohol-seeking behaviors during stressinduced reinstatement (Le et al, 2011) and comorbid depression (Riga et al, 2014) in rats. Collectively, these studies indicate that guanfacine might have the ability to reduce drug use via modulation of craving and dampening of a heightened stress system. However, there is, to our knowledge, no systematic evaluation of guanfacine's ability to attenuate alcohol-related behaviors without the involvement of stress. In addition, guanfacine's ability to affect the glutamatergic system in the PFC following voluntary chronic alcohol consumption needs to be elucidated.

The aim of this study was to evaluate the effect of guanfacine (alone and in comparison with the AUD medication naltrexone) on alcohol-mediated behaviors using a battery of animal models that have shown predictive validity for the clinical population, at least for varenicline (Steensland et al, 2007; McKee et al, 2009; Mitchell et al, 2012; Litten et al, 2013) and the monoamine stabilizer (-)OSU6162 (Steensland et al, 2012, and unpublished data from our research group). Finally, the glutamatergic transmission in the medial PFC (mPFC) was evaluated using electrophysiological recordings in brain slices from long-term drinking rats (and alcohol-naive controls) treated with guanfacine in vivo.

\section{MATERIALS AND METHODS}

Detailed descriptions of the animals (male Rcc Wistar Han Rats (Harlan, The Netherlands)), housing conditions, chemicals, the intermittent-access $20 \%$ ethanol two-bottlechoice (IA20E) paradigm (Wise, 1973; Simms et al, 2008), the operant self-administration paradigms (cue/priminginduced reinstatement and progressive ratio (PR)) (Simms et al, 2010; Steensland et al, 2012), the electrophysiological recordings (Arvanov et al, 1997; Arvanov and Wang, 1998; Konradsson et al, 2006), and descriptions of statistical analyses are available in Supplementary Information.

\section{Treatment Schedules in the Two-Bottle-Choice Experiments}

For the acute guanfacine treatment, 18 rats that had voluntarily consumed alcohol (IA20E) (Wise, 1973; Simms et al, 2008) for $\sim 5$ months were divided into high $(4.3 \pm 0.2 \mathrm{~g} / \mathrm{kg}$ per $24 \mathrm{~h} ; n=11)$ and low alcohol-drinking rats $(1.9 \pm 0.2 \mathrm{~g}$ per $\mathrm{kg} / 24 \mathrm{~h} ; n=7)$ based on their alcohol consumption during the four drinking sessions preceding the first treatment occasion. All rats received all treatments, guanfacine $(0.6$ and $0.3 \mathrm{mg} / \mathrm{kg}$ ) and vehicle, $30 \mathrm{~min}$ before the bottles were presented. All injections were given once a week 7 days apart according to a Latin square design, and thus each rat served as its own control.

For the repeated guanfacine treatment, a second group of rats $(n=12)$ voluntarily consuming high amounts of alcohol $(4.3 \pm 0.2 \mathrm{~g} / \mathrm{kg}$ per $24 \mathrm{~h}$; IA20E) for $\sim 7$ months were divided into two groups with equal alcohol consumption. Thereafter, they received either guanfacine $(0.6 \mathrm{mg} / \mathrm{kg})$ or vehicle treatment $30 \mathrm{~min}$ before five consecutive alcoholdrinking sessions. After the fifth injection, all rats were subjected to a 3-week washout period under regular IA20E drinking. Thereafter, the administration schedule was repeated with treatments reversed between the groups. Thus, each animal served as its own control. Eight alcohol sessions after each treatment period were recorded for measurements of potential rebound increase in alcohol intake.

To compare the efficacy of guanfacine with an established AUD treatment, a third group of rats $(n=11)$ voluntarily consuming high amounts of alcohol $(4.4 \pm 0.2 \mathrm{~g} / \mathrm{kg}$ per $24 \mathrm{~h})$ for $\sim 6$ months (IA20E), were given guanfacine $(0.6 \mathrm{mg} / \mathrm{kg})$, naltrexone $(2 \mathrm{mg} / \mathrm{kg})$, or vehicle according to a Latin square design as described for the acute guanfacine experiments above. The naltrexone dose was chosen based on previous dose-response studies in the IA20E model (Simms et al, 2008).

To evaluate the ability of guanfacine to decrease voluntary alcohol intake after a period of protracted abstinence, we used the alcohol deprivation model that is based on the observation that voluntary alcohol intake will increase temporarily (compared with baseline) when alcohol is reintroduced after a period of forced abstinence in alcohol-experienced rats (ie, the alcohol deprivation effect (ADE)) (Spanagel and Holter, 2000). Two groups of rats were alcohol-deprived during 17 days after at least 8 weeks of voluntary consumption of high $(4.7 \pm 0.2 \mathrm{~g} / \mathrm{kg}$ per $24 \mathrm{~h})$ or moderate $(2.6 \pm 0.2 \mathrm{~g} / \mathrm{kg}$ per $24 \mathrm{~h})$ amounts of alcohol (IA20E), respectively. Each group was thereafter divided into three subgroups with equal alcohol intake during the last three drinking sessions, and subjected to vehicle (saline), guanfacine $(0.6 \mathrm{mg} / \mathrm{kg})$, or naltrexone $(2 \mathrm{mg} / \mathrm{kg})$ treatment ( $n=6-9$ per group) $60 \mathrm{~min}$ before alcohol was reintroduced. The alcohol intake was measured after 4 and $24 \mathrm{~h}$ of alcohol access.

The effect of guanfacine on NMDA- and AMPA-induced currents in pyramidal cells in layer V or VI of the prelimbic cortex in mPFC was evaluated in the long-term drinking rats from the $\mathrm{ADE}$ experiment. After the $\mathrm{ADE}$ experiment, each rat was given at least 1 week of alcohol consumption before receiving three daily consecutive injections (i.p.) of either guanfacine $(0.6 \mathrm{mg} / \mathrm{kg}, n=7)$ or vehicle $(n=5)$. Each rat received the same treatment (guanfacine or vehicle) as in the ADE experiment. The brains were collected $24 \mathrm{~h}$ after the last injection and slices were prepared as described previously (Arvanov et al, 1997; Konradsson et al, 2006). As a control, two groups of alcohol-naive age-matched rats underwent the same guanfacine or vehicle treatment as above ( $n=5-6$ per group). Details of the electrophysiological recordings are found in Supplementary Information.

The ability of guanfacine $(0.6 \mathrm{mg} / \mathrm{kg})$ to decrease alcohol intake without inducing sedative effects was evaluated in two experiments. (1) Acute guanfacine or vehicle treatment was given to rats $(n=8)$ that had consumed $0.175 \% \mathrm{NaCl}$ solution for 7 months (intermittent-access two-bottle-choice schedule) as we have previously shown that compounds that specifically decrease the intake of reinforcing solutions, such as alcohol, saccharine, or sucrose, have no effect on the intake of a salty solution (Steensland et al, 2010; Steensland 
et al, 2012). All rats got both guanfacine and vehicle treatments using a within-subject design with 7 days between each injection. (2) Three daily guanfacine or vehicle injections were given to alcohol-naive rats (same rats as in the electrophysiological experiment) and the effect on the water intake was measured $23 \mathrm{~h}$ after each injection $(n=5-6$ rats per treatment group).

\section{RESULTS}

Acute Guanfacine Treatment Decreased Alcohol Intake in Rats Voluntarily Consuming High Amounts of Alcohol

The effects of acute guanfacine treatment $(0.3$ and $0.6 \mathrm{mg} / \mathrm{kg})$ on voluntary alcohol consumption was evaluated in a group of rats that had voluntarily emerged into high $(4.3 \pm 0.2 \mathrm{~g} / \mathrm{kg}$ per $24 \mathrm{~h} ; n=11)$ or low $(1.9 \pm 0.2 \mathrm{~g} / \mathrm{kg}$ per $24 \mathrm{~h} ; n=7)$ alcohol consumers following $\sim 5$ months of IA20E. Analysis of the alcohol intake showed an overall main effect of treatment $(4 \mathrm{~h}(\mathrm{~F}(3,48)=18, P<0.001)$ and $24 \mathrm{~h}(\mathrm{~F}(3,48)=$ $18, P<0.001))$ and type (high or low drinkers) at both time points $(4 \mathrm{~h}(\mathrm{~F}(1,16)=36, P<0.001)$ and $24 \mathrm{~h}(\mathrm{~F}(1,16)=33$, $P<0.001)$ ). Furthermore, there was a significant interaction of treatment $\times$ type $(4 \mathrm{~h}(\mathrm{~F}(3,48)=6.7, P<0.01)$ and $24 \mathrm{~h}$ $(\mathrm{F}(3,48)=5.0, P<0.01))$. The post hoc analysis revealed that both doses of guanfacine significantly decreased voluntary alcohol intake compared with vehicle in the high-alcoholconsuming rats (Figure 1a). However, there was no significant effect on the alcohol intake following guanfacine treatment in the rats consuming low amounts of alcohol (Figure 1b). Furthermore, there was a significant decrease in alcohol preference in the rats consuming high, but not low, amounts of alcohol (treatment: $4 \mathrm{~h}(\mathrm{~F}(3,48)=5.0, P<0.01)$ and $24 \mathrm{~h}((3,48)=31, P<0.001)$; type: $4 \mathrm{~h}(\mathrm{~F}(1,16)=21$, $P<0.001)$ and $24 \mathrm{~h}(\mathrm{~F}(1,16)=21, P<0.001)$; treatment $\times$ type: $4 \mathrm{~h}(\mathrm{~F}(3,48)=3.2, P<0.05)$ and $24 \mathrm{~h}(\mathrm{~F}(3,48)=6.1$, $P<0.001)$; Supplementary Table S1), whereas there was no significant effect on the water intake in either group (treatment: $4 \mathrm{~h}(\mathrm{~F}(3,48)=2.0$, nonsignificant (n.s.)) and $24 \mathrm{~h} \quad \mathrm{~F}(3,48)=32, \quad P<0.001)$; type: $4 \mathrm{~h} \quad(\mathrm{~F}(1,16)=9.1$, $P<0.01)$ and $24 \mathrm{~h}(\mathrm{~F}(1,16)=5.3, P<0.05)$; treatment $\times$ type: $4 \mathrm{~h}(\mathrm{~F}(3,48)=1.9$, n.s. $)$ and $24 \mathrm{~h}(\mathrm{~F}(3,48)=1.2$, n.s. $)$; Supplementary Table S1). When analyzing the alcohol intake during the first drinking session after the treatment in the rats consuming high amounts of alcohol, there was no overall main effect at any time point $(4 \mathrm{~h}(\mathrm{~F}(2,32)=0.2$, n.s.) and $24 \mathrm{~h}(\mathrm{~F}(2,32)=0.06$, n.s.); Figure 1c). Thus, no post hoc analysis was performed. Finally, guanfacine treatment had no significant effect on voluntary intake of a salty solution compared with vehicle (Supplementary Table S2).

\section{Repeated Guanfacine Treatment Induced Long-Lasting Decrease in Voluntary Alcohol Intake}

The effects of repeated guanfacine treatment $(0.6 \mathrm{mg} / \mathrm{kg} / \mathrm{day})$ during five consecutive drinking sessions were evaluated in rats that had voluntarily consumed high amounts of alcohol $(4.3 \pm 0.2 \mathrm{~g} / \mathrm{kg}$ per $24 \mathrm{~h})$ for $\sim 7$ months. When analyzing the effects on alcohol intake at the 4 and $24 \mathrm{~h}$ time points (Figure 2), there was an overall main effect on treatment
$(4 \mathrm{~h}(\mathrm{~F}(1,11)=144, P<0.0001)$ and $24 \mathrm{~h}(\mathrm{~F}(1,11)=137$, $P<0.0001))$, day $(4 \mathrm{~h}(\mathrm{~F}(12,132)=9.7, P<0.0001)$ and $24 \mathrm{~h}$ $(\mathrm{F}(12,132)=13, P<0.0001))$, and a significant interaction of treatment $\times$ day $(4 \mathrm{~h}(\mathrm{~F}(12,132)=11, P<0.0001)$ and $24 \mathrm{~h} \quad(\mathrm{~F}(12,132)=15, P<0.0001))$. Planned comparisons revealed that guanfacine significantly decreased voluntary alcohol intake compared with vehicle on all five treatment days and remained significantly lower compared with vehicle for up to five alcohol drinking sessions after the guanfacine treatment was terminated. In addition, there was an overall main effect at both time points for alcohol preference for treatment $(4 \mathrm{~h}(\mathrm{~F}(1,11)=72, P<0.0001)$ and $24 \mathrm{~h}(\mathrm{~F}(1,11)=99, P<0.0001))$, day $(4 \mathrm{~h}(\mathrm{~F}(12,132)=7.0$, $P<0.0001)$ and $24 \mathrm{~h}(\mathrm{~F}(12,132)=20, P<0.0001))$, and $\mathrm{a}$ significant interaction of treatment $\times$ day $(4 \mathrm{~h}(\mathrm{~F}(12,132)=$ 5.4, $P<0.0001)$ and $24 \mathrm{~h}(\mathrm{~F}(12,132)=17, P<0.0001))$ as well as for the water intake (treatment: $4 \mathrm{~h}(\mathrm{~F}(1,11)=21$, $P=0.001)$ and $24 \mathrm{~h} \quad(\mathrm{~F}(1,11)=90, P<0.0001) ;$ day: $4 \mathrm{~h}$ $(\mathrm{F}(12,132)=2.4, \quad P<0.01) \quad$ and $\quad 24 \mathrm{~h} \quad(\mathrm{~F}(12,132)=20$, $P<0.0001)$; treatment $\times$ day: $4 \mathrm{~h}(\mathrm{~F}(12,132)=1.9, P<0.05)$ and $24 \mathrm{~h}(\mathrm{~F}(12,132)=15, P<0.0001))$. Planned comparisons showed that guanfacine significantly decreased alcohol preference and increased water intake compared with vehicle. Furthermore, there was no overall main effect of treatment $(\mathrm{F}(1,9)=0.02$, n.s. $)$ or day $(\mathrm{F}(3,27)=0.9$, n.s. $)$ on the water intake in the alcohol-naive rats that received three consecutive guanfacine or vehicle injections (Supplementary Table S3).

\section{Guanfacine Was More Efficacious Than Naltrexone to Decrease Alcohol Intake in Rats Voluntarily Consuming High Amounts of Alcohol}

The efficacy of guanfacine $(0.6 \mathrm{mg} / \mathrm{kg})$ to attenuate voluntary alcohol intake was compared with that of the AUD medication naltrexone $(2 \mathrm{mg} / \mathrm{kg})$ in rats that had voluntary consumed high amounts of alcohol $(4.4 \pm 0.2 \mathrm{~g} / \mathrm{kg}$ per $24 \mathrm{~h}$; $n=11)$ for $\sim 6$ months before the treatment. There was an overall main effect on alcohol intake at both the $4 \mathrm{~h}$ $(\mathrm{F}(2,20)=40, P<0.0001)$ and $24 \mathrm{~h}(\mathrm{~F}(2,20)=60, P<0.0001)$ time points. Planned comparisons revealed that both guanfacine and naltrexone significantly decreased voluntary alcohol intake compared with vehicle at the $4 \mathrm{~h}$ time point (Figure 3a, left panel), whereas only guanfacine significantly decreased the intake compared with vehicle at the $24 \mathrm{~h}$ time point (Figure 3a, right panel). In addition, guanfacine significantly decreased alcohol intake compared with naltrexone at both time points.

In the ADE experiment, two groups of rats were subjected to a forced alcohol abstinence period after at least 8 weeks of voluntary intake of moderate or high amounts of alcohol, respectively. Following the reintroduction of alcohol, the vehicle-treated rats drinking moderate (Figure 3b), but not high (Figure 3c), amounts of alcohol significantly increased their alcohol intake at both time points compared with corresponding baseline before the abstinence period. Both guanfacine and naltrexone significantly decreased alcohol intake after the abstinence period compared with respective baseline in both moderate (Figure $3 \mathrm{~b}$ ) and high (Figure 3c) drinkers. The effect of guanfacine was more long lasting than that of naltrexone, especially in high drinkers. 


\section{Acute Guanfacine Treatment}

a

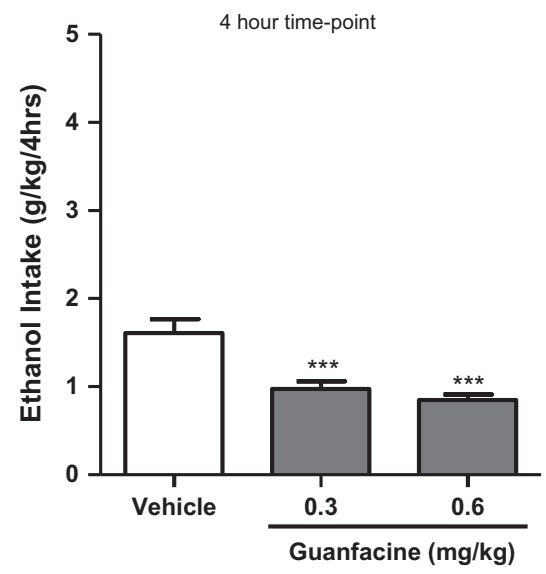

High Drinkers

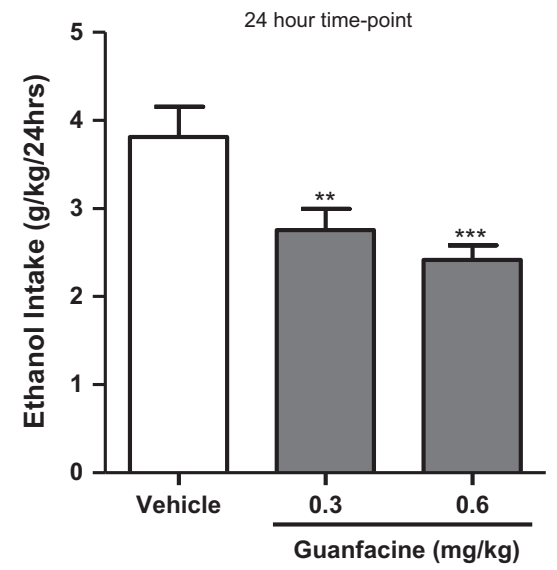

b

Low Drinkers
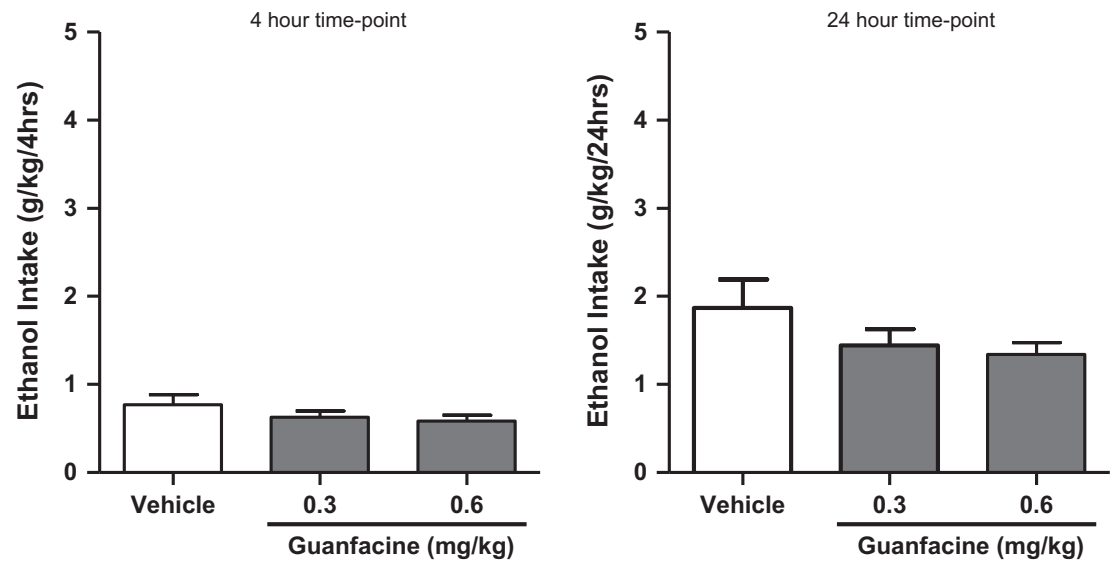

C

High Drinkers - First Drinking Session After Treatment
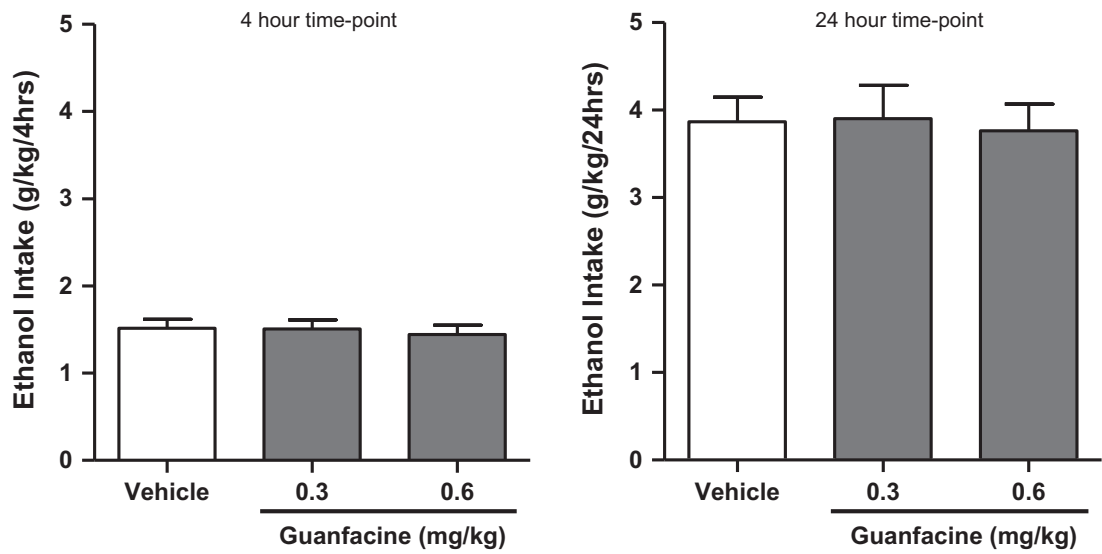

Figure I Acute guanfacine treatment $(0.3$ and $0.6 \mathrm{mg} / \mathrm{kg})$ significantly decreased voluntary alcohol intake compared with vehicle in rats voluntarily consuming (a) high $(4.3 \pm 0.2 \mathrm{~g} / \mathrm{kg}$ per $24 \mathrm{~h} ; n=1 \mathrm{I})$ but not (b) low $(1.9 \pm 0.2 \mathrm{~g} / \mathrm{kg}$ per $24 \mathrm{~h} ; n=7)$ amounts of alcohol for $\sim 5$ months before the treatment. (c) There was no rebound increase in alcohol intake during the first drinking session after treatment in rats consuming high amounts of alcohol. All values are expressed as mean \pm SEM; **P $<0.01$, ***P $<0.00$ I compared with corresponding vehicle $((a, b)$ two-way repeated-measures ANOVA followed by planned comparisons; (c) one-way repeated-measures ANOVA followed by Newman-Keuls multiple comparison test). 

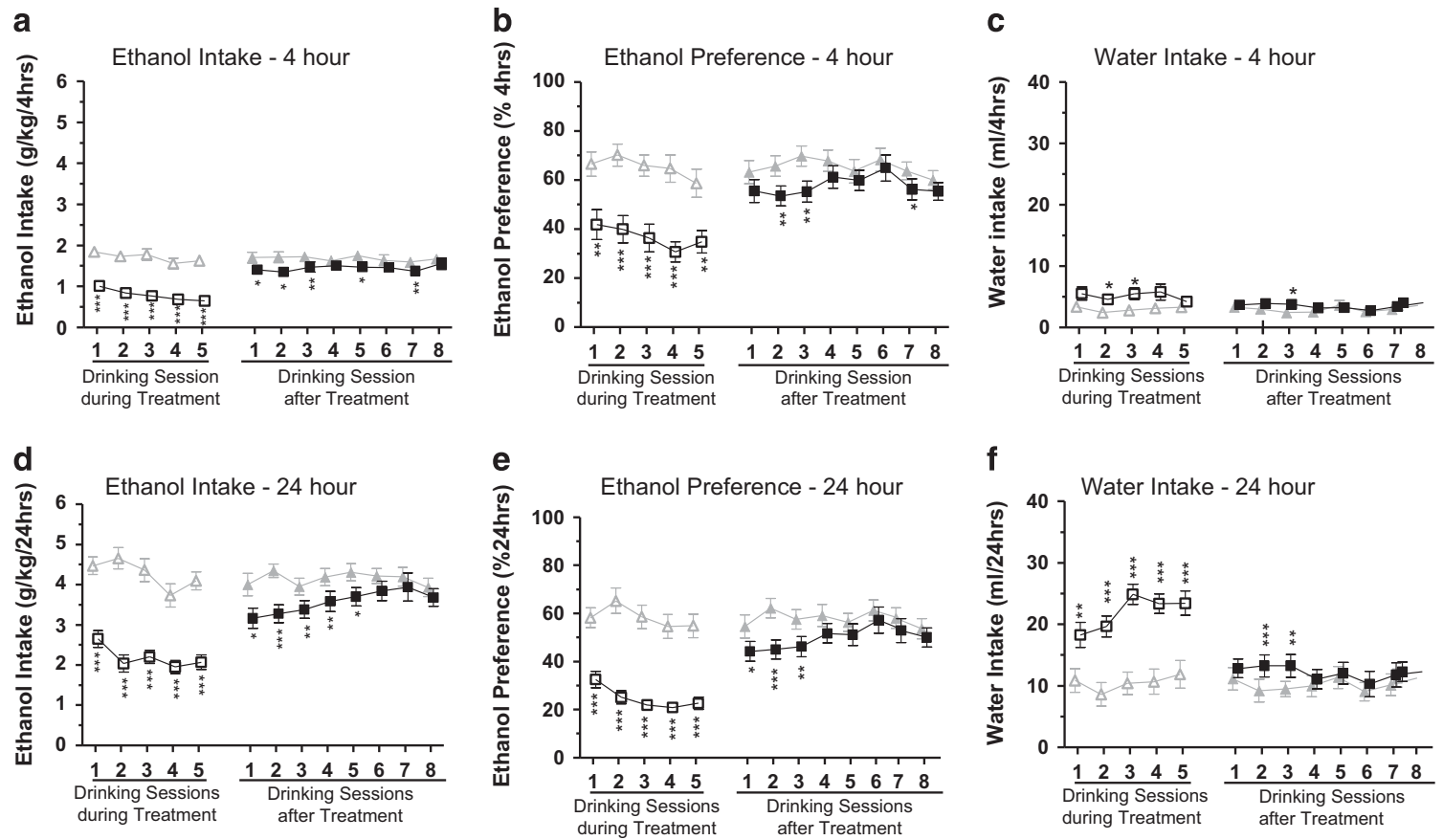

\begin{tabular}{|c|c|c|c|}
\hline$\triangle-$ Vehicle & 曰 Guanfacine $(0.6 \mathrm{mg} / \mathrm{kg})$ & - Previously Vehicle Treated & $\rightarrow-$ Previously Guanfacine Tre \\
\hline
\end{tabular}

Figure 2 Repeated guanfacine treatment $(0.6 \mathrm{mg} / \mathrm{kg} /$ day over 5 consecutive alcohol drinking sessions) significantly decreased voluntary alcohol intake ((a) $4 \mathrm{~h}$ time point and (d) $24 \mathrm{~h}$ time point) and preference for alcohol ((b) $4 \mathrm{~h}$ time point and (e) $24 \mathrm{~h}$ time point) compared with vehicle in rats that had been consuming high amounts of alcohol $(4.3 \pm 0.2 \mathrm{~g} / \mathrm{kg}$ per $24 \mathrm{~h})$ for $\sim 7$ months before the treatment. There was no tolerance development during treatment and the significant decrease persisted for up to five drinking sessions after the last injection. In addition, there was a significant increase in the water intake ((c) $4 \mathrm{~h}$ time point and (f) $24 \mathrm{~h}$ time point) compared with vehicle. All values are expressed as mean $\pm S E M ; n=\mid 2 ; * P<0.05$, *** $P<0.01$, **** $P<0.00$ I compared with corresponding vehicle (two-way repeated-measures ANOVA followed by planned comparisons).

\section{Guanfacine Significantly Attenuated Alcohol Seeking and Cue/Priming-Induced Reinstatement of Alcohol-Seeking Behavior}

The effect of guanfacine on alcohol-seeking behavior was evaluated using the PR test in rats that had been voluntarily exposed to alcohol for $\sim 5$ months using operant selfadministration (see details in Supplementary Information). There was an overall main effect on active lever presses $(\mathrm{F}(2,24)=16, P<0.0001)$, breakpoint $(\mathrm{F}(2,24)=28, P<0.0001)$, and inactive lever presses $(\mathrm{F}(2,24)=6.5, P<0.01)$. The post hoc analysis shows that both guanfacine doses significantly inhibited the number of active lever presses as well as the breakpoint compared with vehicle (Figure 4a). The higher guanfacine dose significantly $(P=0.01)$ decreased the number of inactive lever presses compared with vehicle (vehicle: $2.0 \pm 0.5$; guanfacine $(0.3 \mathrm{mg} / \mathrm{kg}): 1.3 \pm 0.4$; guanfacine $(0.6 \mathrm{mg} / \mathrm{kg}): 0.5 \pm 0.2)$.

After the PR test and 2 weeks of baseline self-administration of alcohol, the alcohol-seeking behavior was extinguished during 16 extinction sessions (without the presence of olfactory, visual, and auditory cues) where pressing the active lever produced no delivery of alcohol. During the reinstatement session (where the cues together with a small amount of alcohol in the liquid dispenser (equivalent to one reward) were reintroduced), there was an overall main effect on the number of active lever presses $(\mathrm{F}(3,30)=11, P<0.001)$. The post hoc analysis revealed that the vehicle pretreated group reinstated (ie, significantly increased the number of active lever presses compared with that during extinction) and that guanfacine pretreatment attenuated this response (Figure $4 \mathrm{~b}$, left panel). There was no overall main effect on the number of inactive lever presses $(F(3,30)=0.4$, n.s.) (Figure $4 \mathrm{~b}$, right panel).

Guanfacine Normalized Alcohol-Induced Dysregulated Glutamatergic Neurotransmission in Pyramidal Cells of the MPFC

Guanfacine $(0.6 \mathrm{mg} / \mathrm{kg} /$ day, i.p.) or vehicle was given during three consecutive days to two groups of rats that had voluntarily been drinking high amounts of alcohol for at least 11 weeks. Alcohol-naive age-matched controls underwent the same treatment. The electrophysiology recordings were performed in vitro (brains were collected $24 \mathrm{~h}$ after the last injection) on cells located in layers V and VI of the prelimbic cortex in the mPFC (located medial to the forceps minor and easily identified in a slice). The electrophysiological criteria used for distinguishing presumed pyramidal versus nonpyramidal cells are described elsewhere (Arvanov et al, 1997; Konradsson et al, 2006). In general, the pyramidal cells exhibited longer spike duration $(>1 \mathrm{~ms}$ at 

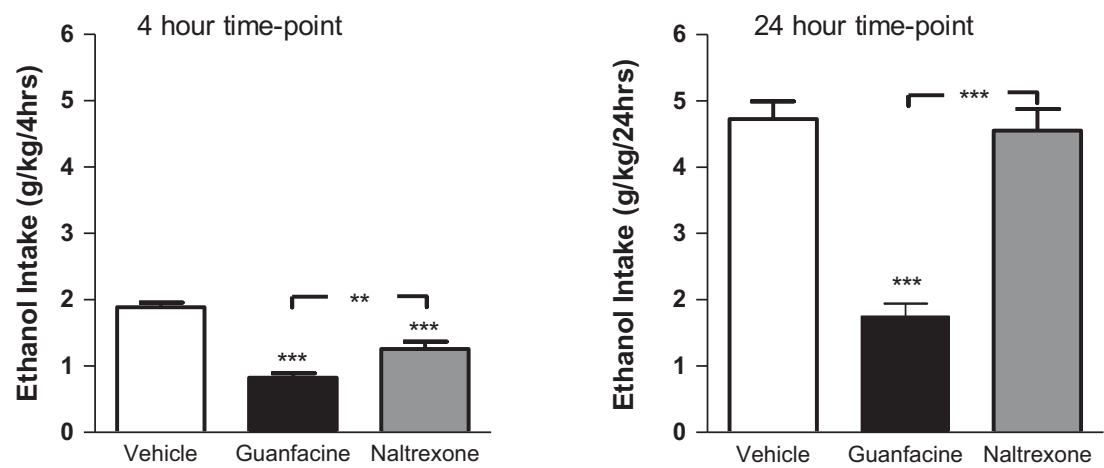

b Alcohol Deprivation Effect - Moderate Drinkers
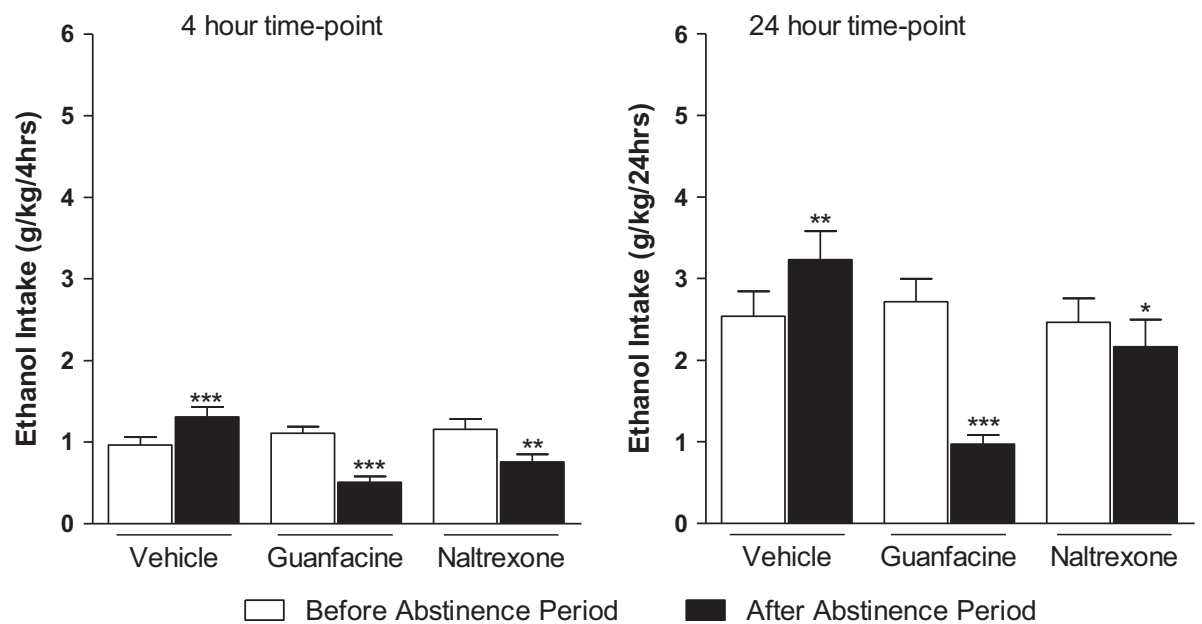

C

Alcohol Deprivation Effect - High Drinkers
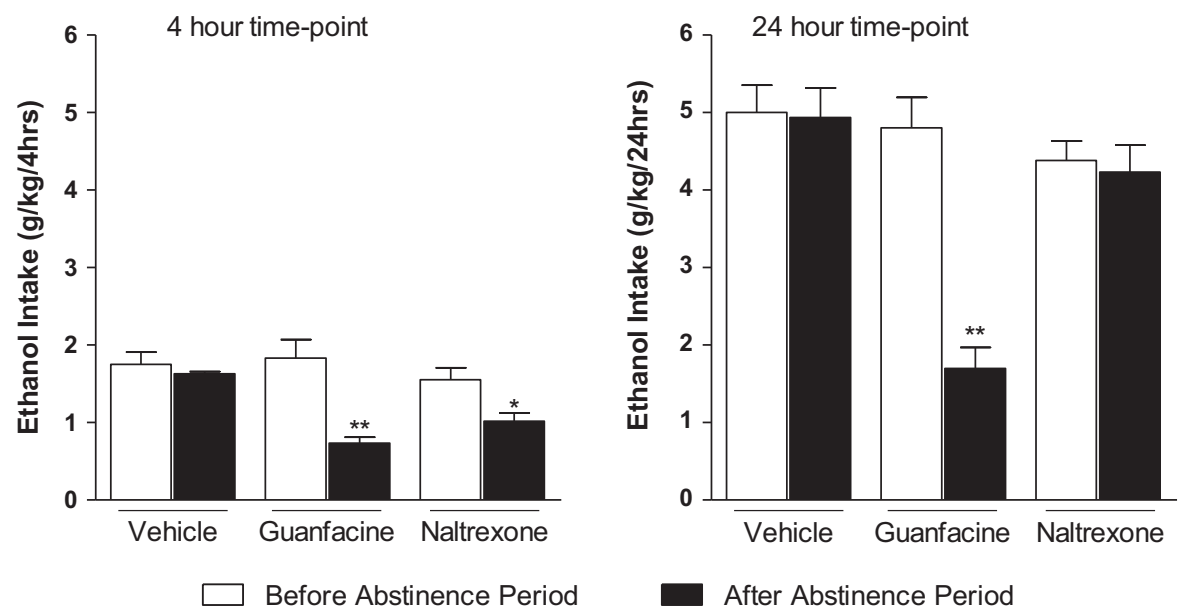

Figure 3 Both guanfacine $(0.6 \mathrm{mg} / \mathrm{kg})$ and naltrexone $(2 \mathrm{mg} / \mathrm{kg}$ ) significantly decreased alcohol intake compared with vehicle at the $4 \mathrm{~h}$ time point (a, left panel) in rats that had voluntarily consumed high amounts of alcohol for $\sim 6$ months before the treatment $(n=1 \mathrm{l})$. At the $24 \mathrm{~h}$ time point $(\mathrm{a}$, right panel) only guanfacine significantly decreased the alcohol intake compared with vehicle. At both time points, guanfacine significantly decreased the alcohol intake compared with naltrexone. All values are expressed as mean $\pm \mathrm{SEM}$; $* * P<0.0$ I, $* * * P<0.00$ I compared with vehicle or as indicated (one-way repeatedmeasures ANOVA followed by planned comparisons). To evaluate the effect of guanfacine and naltrexone on the alcohol deprivation effect (ADE), two groups of rats voluntarily consuming (b) moderate $(2.6 \pm 0.2 \mathrm{~g} / \mathrm{kg}$ per $24 \mathrm{~h})$ or (c) high $(4.7 \pm 0.2 \mathrm{~g} / \mathrm{kg}$ per $24 \mathrm{~h})$ amounts of alcohol for at least 8 weeks were subjected to a period of 17 days with forced alcohol abstinence before alcohol was reintroduced. The rats were pretreated with guanfacine $(0.6 \mathrm{mg} / \mathrm{kg})$, naltrexone $(2 \mathrm{mg} / \mathrm{kg})$, or vehicle before renewed alcohol access $(n=6-9$ per treatment group). An ADE was present in vehicle-treated rats consuming moderate (b) but not high (c) amounts of alcohol. Both guanfacine- and naltrexone-treated rats significantly decreased their alcohol intake in both high (c) and moderate (b) drinkers compared with corresponding baseline before the abstinence period, with the effects of guanfacine being more pronounced and long lasting than naltrexone. All values are expressed as mean $\pm \mathrm{SEM}$; $* P<0.05$, $* * P<0.0$ I, $* * * P<0.00$ I compared with corresponding values before the abstinence period (paired Student's t-test within each treatment). 
a

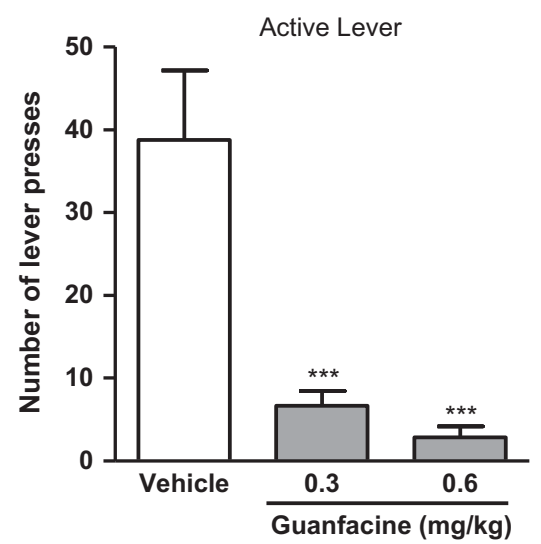

Progressive Ratio

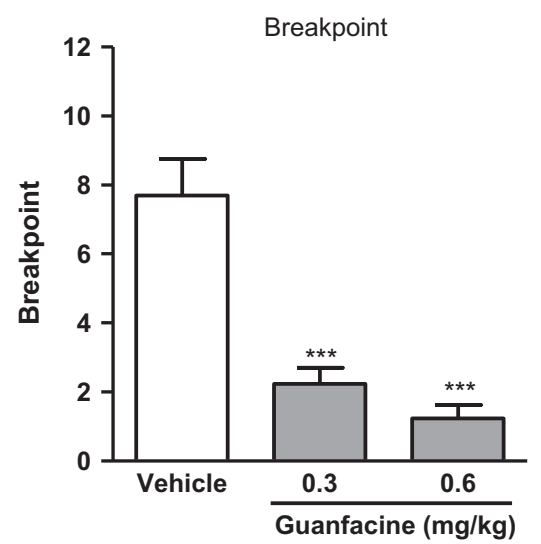

b

Cue/Priming-Induced Reinstatement

Active Lever

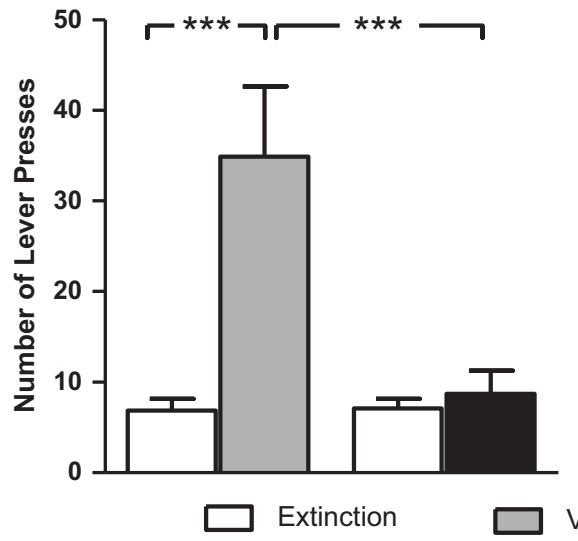

Inactive Lever

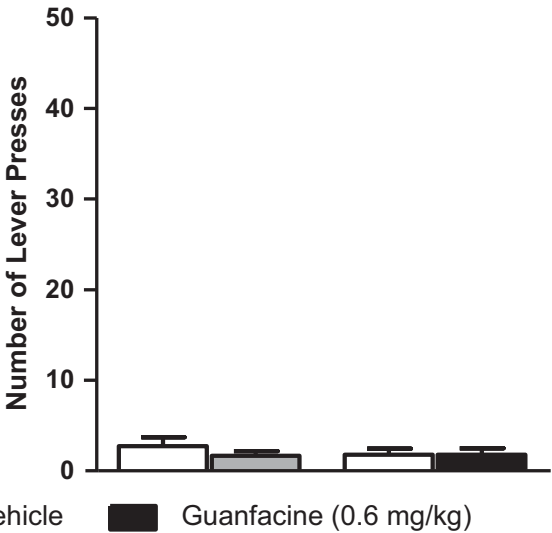

Figure 4 The effect of guanfacine on alcohol-seeking behavior was evaluated using the progressive ratio (PR) test (a) in rats that had been voluntarily exposed to alcohol for $\sim 5$ months using operant self-administration (see details in Supplementary Information). Both guanfacine doses (0.3 and $0.6 \mathrm{mg} / \mathrm{kg}$ ) significantly decreased the number of presses on the active lever (a, left panel) as well as the breakpoint for alcohol (a, right panel) compared with vehicle. Each rat received all doses and vehicle according to a Latin square design over a 3-week period with 7 days between each injection. The values are expressed as mean $\pm \mathrm{SEM} ; n=13$; **** $<0.001$ compared with vehicle (one-way repeated-measures ANOVA followed by planned comparisons with adjustments for multiple comparisons). After the PR test, and 2 weeks of baseline responding on FR3, the alcohol-seeking behavior of rats was extinguished during 16 extinction sessions where pressing the active lever produced no delivery of alcohol. The extinction sessions were conducted without the presence of olfactory, visual, and auditory cues. Before the start of the reinstatement session, half of the rats received guanfacine $(0.6 \mathrm{mg} / \mathrm{kg})$ or vehicle $60 \mathrm{~min}$ before the start of the reinstatement session, during which the cues previously associated with the delivery of alcohol were reintroduced. (b, left panel) The vehicle-treated animals reinstated the alcohol-seeking behavior as shown by a significant increase in the number of active lever press compared with the extinction level. The reinstatement response was significantly attenuated by guanfacine treatment (b, left panel). There was no significant effect on the number of lever presses on the inactive lever (b, right panel). All values are presented as mean \pm SEM; vehicle $(n=8)$; guanfacine $(n=9)$; *** $P<0.001$ as indicated in the figure (one-way ANOVA followed by Newman-Keuls multiple comparison test).

half-maximum spike amplitude) than interneurons, and showed pronounced spike-frequency adaptation in response to constant-current depolarizing pulses. In contrast, interneurons exhibited a brief duration of their action potentials and generally lacked pronounced spike-frequency adaptation. There was an overall main effect on both NMDA-induced $(\mathrm{F}(3,19)=22, P=0.0001$; Figure 5a) and AMPA-induced $(\mathrm{F}(3,19)=30, P<0.0001$; Figure $5 \mathrm{~b})$ inward currents in pyramidal cells of mPFC slices. The post hoc analysis revealed that long-term drinking rats had a significant increase in both NMDA and AMPA currents compared with the alcohol-naive rats. The in vivo guanfacine treatment significantly reversed this alcohol-induced dysregulation, whereas there was no significant effect of guanfacine in the alcohol-naive rats.

\section{DISCUSSION}

The main findings in this study were that guanfacine, a FDA-approved ADHD medication, attenuated voluntary alcohol consumption and relapse behavior in Wistar rats that had voluntarily consumed alcohol for at least 2 months before treatment. The effects of guanfacine were generally more pronounced and long lasting than that of the AUD medication naltrexone. In fact, repeated guanfacine 
a

NMDA -Induced Currents

A

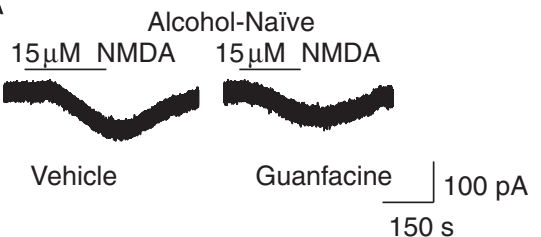

B

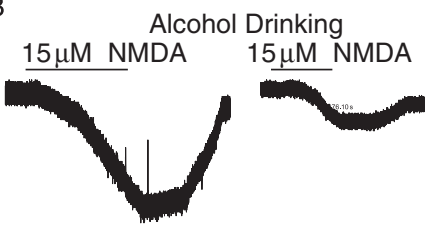

Vehicle

Guanfacine $100 \mathrm{pA}$

$150 \mathrm{~s}$

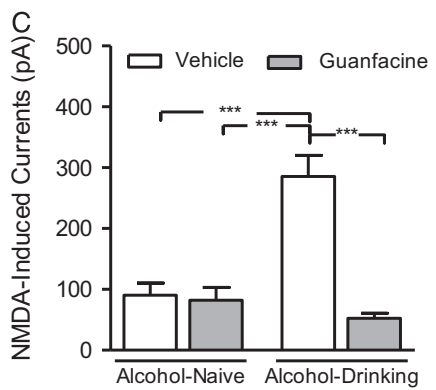

b

AMPA -Induced Currents

A Alcohol-Naïve

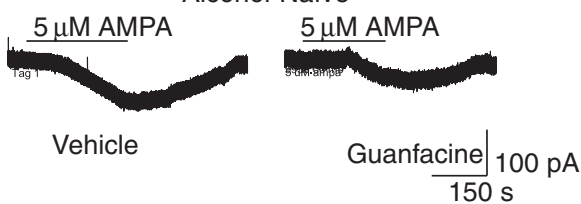

B

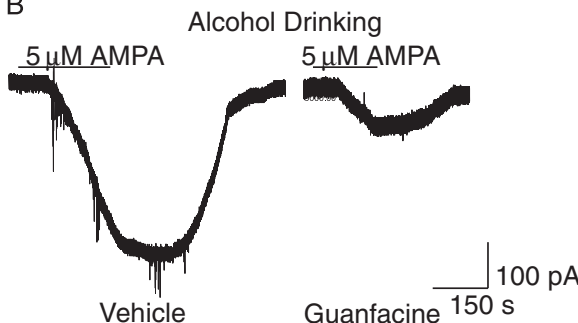

C

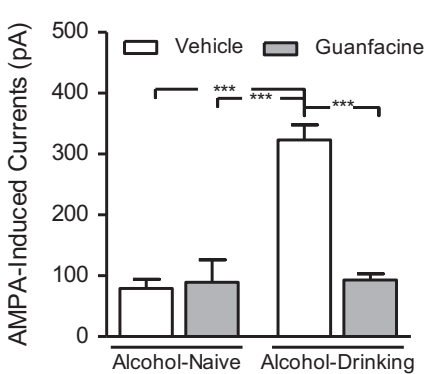

Figure 5 The electrophysiology recordings were conducted in vitro in pyramidal cells in layer $\mathrm{V}$ or $\mathrm{VI}$ in slices prepared from the prelimbic cortex of the $\mathrm{mPFC}$ of rats that had voluntarily been drinking high amounts of alcohol for at least I I weeks. Before the experiment the rats received three daily guanfacine $(0.6 \mathrm{mg} / \mathrm{kg} /$ day, i.p.) or vehicle injections in vivo. Brains were collected $24 \mathrm{~h}$ after the last injection. Alcohol-naïve, age-matched rats were used as controls. The long-term alcohol consumption significantly increased both the NMDA- (a) and AMPA-induced (b) currents compared with alcohol-naïve controls. Guanfacine treatment normalized this alcohol-evoked facilitation while having no significant effect in the alcohol-naïve rats. The holding potential was $-60 \mathrm{mV}$. Representative traces showing the NMDA- and AMPA-induced currents following guanfacine and vehicle treatment in alcohol-naïve and alcohol-drinking-rats are shown in $(A)$ and $(B)$, respectively. The summarized data (mean $\pm S E M)$ are found in $(C) ; n=6$ cells per treatment in the longterm-drinking rats and $n=4$ cells per treatment in the alcohol-naïve rats; $* * * P<0.000$ I as indicated in the figure (one-way ANOVA, Newman-Keuls multiple comparison test).

treatment induced long-lasting reduction in voluntary alcohol intake even when the medication was no longer on board. In addition, we show, to our knowledge for the first time, that in vivo guanfacine treatment normalized dysregulated glutamatergic neurotransmission in the $\mathrm{mPFC}$, induced by long-term voluntary alcohol consumption.

In this study, guanfacine demonstrated several desirable effects of a potential AUD medication. First, repeated guanfacine treatment significantly decreased alcohol intake in rats voluntarily consuming high amounts of alcohol, without tolerance development to guanfacine's effect. Second, the alcohol intake was significantly reduced compared with vehicle for up to five drinking sessions (equivalent to 12 days given the IA20E schedule) after termination of the repeated, but not acute, guanfacine treatment, indicating that sustained guanfacine treatment might induce longlasting neurochemical alterations beneficial for maintenance of reduced alcohol consumption. Finally, guanfacine decreased voluntary alcohol intake after a long period of forced abstinence as well as alcohol seeking under PR schedule and cue/priming-induced reinstatement. The role of guanfacine as a relapse prevention agent is further supported by previous animal and human studies showing that the compound attenuates stress-induced relapse of alcohol and cocaine (Le et al, 2011; Fox et al, 2012, 2014) and the motivational drive to seek alcohol in depressed rats (Riga et al, 2014).

The electrophysiological experiment in this study showed that guanfacine can normalize alcohol-induced dysregulation of the glutamatergic neurotransmission in the mPFC. These results reflect an in vivo pharmacologically induced response in the $\mathrm{mPFC}$ as the brain slices were prepared from long-term drinking rats that were treated with three daily guanfacine injections $(0.6 \mathrm{mg} / \mathrm{kg} /$ day $)$ before the experiment. Together with guanfacine's promising effects on alcohol-mediated behaviors (presented above), these electrophysiology results support the hypothesis that the glutamatergic system might be an important treatment target for AUD (Holmes et al, 2013).

The mechanism underlying guanfacine's ability to modulate the glutamatergic system in AUD is not fully understood. Chronic alcohol consumption has an inhibitory effect on both NMDA and AMPA receptors that in turn have been suggested to produce adaptive changes with an increased 
expression of subunits of these receptors (see review (Holmes et al, 2013)). Such alcohol-induced dysregulation is supported by the present findings, showing increased NMDA- and AMPA-induced currents in pyramidal cells in the $\mathrm{mPFC}$ in rats that had consumed high amounts of alcohol for $\sim 3$ months. Interestingly, 3 days of guanfacine treatment normalized this alcohol-induced hyperglutamatergic transmission, providing support for a role of this $\alpha$-2-adrenoceptor agonist as a modulator of the glutamatergic system in the mPFC. In fact, guanfacine has previously been shown to inhibit the production of cAMP, with related blockade of cAMP-dependent and hyperpolarization-activated cyclic nucleotide-gated channels leading to enhanced excitatory transmission and improved PFC connectivity (Wang et al, 2007). This mechanism together with previous studies showing that guanfacine improves impulsive behavior in rats (Fernando et al, 2012) as well as working memory (with corresponding increase in blood flow in the PFC) in monkeys (Avery et al, 2000) strengthen the case of guanfacine's therapeutic profile as a cognitive enhancer.

In this study it was found that the effects of guanfacine were selective to high alcohol consumption as shown by: (1) a significant effect of guanfacine on the NMDA- and AMPAinduced currents in long-term drinking, but not alcoholnaive rats and (2) reduced alcohol intake in rats that had voluntarily been drinking higher, but not lower, amounts of alcohol for $\sim 5$ months before the treatment. This selective effectiveness is supported by previous clinical studies indicating that guanfacine's therapeutic efficacy may be more specific in conditions characterized by dysfunction in the catecholamine systems (Arnsten et al, 1996) such as ADHD and impulse control disorders (Taylor and Russo, 2001). Indeed, alcohol and substance use disorders share common pathophysiology with ADHD (including PFC dysregulation) (Goldstein and Volkow, 2011) and the present results indicate that guanfacine might have the ability to target and strengthen the neuronal network in mPFC following long-term alcohol consumption, in addition to the documented effects in ADHD (Sallee et al, 2009a, b; Sallee and Eaton, 2010).

In this study, both guanfacine and the AUD medication naltrexone significantly decreased voluntary alcohol intake in long-term-drinking rats. However, our results indicate that guanfacine might be superior to naltrexone. For example, the effect of guanfacine was more pronounced and long lasting than naltrexone, both during an ongoing alcohol consumption period and during the first drinking session following a protracted abstinence period. However, a study comparing several guanfacine and naltrexone doses are needed to confirm these findings. Guanfacine might also have beneficial effect for a wider clinical population than naltrexone. Naltrexone is mostly efficacious in AUD patients with a specific polymorphism in the $\mu$-opioid receptor gene (Oslin et al, 2003; Anton et al, 2008). Guanfacine, on the other hand, modulates both central and peripheral noradrenergic pathways (van Zwieten, 1999; Sica, 2007; Arnsten, 2011), regulating a variety of alcohol-related mood and behavioral states and could thus play a salient role in the treatment of not only AUD per se but also comorbid anxiety, depression, and stress-related compulsive drug use (Le et al, 2011; Fox et al, 2012; Riga et al, 2014).
In rats, an increased demand for alcohol after protracted abstinence (ie, the ADE) has been suggested to be induced by cues (eg, the smell of alcohol) or a priming stimulus (eg, intake of small amount of alcohol) (Spanagel and Holter, 2000) and to be dissociated from normal eating or drinking behavior (Sanchis-Segura and Spanagel, 2006). Thus, the $\mathrm{ADE}$ has been suggested to resemble a typical relapse situation in AUD patients. In this study, guanfacine and naltrexone significantly attenuated ADE occurring after vehicle treatment in rats voluntarily consuming moderate amounts of alcohol $(2.6 \pm 0.2 \mathrm{~g} / \mathrm{kg}$ per $24 \mathrm{~h})$. In contrast, the vehicle-treated rats consuming high amounts of alcohol $(4.7 \pm 0.2 \mathrm{~g} / \mathrm{kg}$ per $24 \mathrm{~h})$ did not display an ADE. Nevertheless, both guanfacine and naltrexone decreased alcohol intake compared with corresponding baseline before the abstinence period also in the high-alcohol-consuming rats. The lack of ADE in rats voluntary drinking high amounts of alcohol might be because of a ceiling level in alcohol intake. This hypothesis is supported by a previous study showing that AA rat selectively bred for high alcohol preference (consuming similar levels of alcohol as the high-alcoholconsuming Wistar rats in the present study) do not display an ADE (Sinclair and Tiihonen, 1988). However, it should be noted that an ADE has been found in other rat strains selectively bred for high alcohol preference (McKinzie et al, 1998; Vengeliene et al, 2003), indicating that other factors, in addition to the level of baseline consumption, are important for the induction of an ADE. Regardless of the presence of an $\mathrm{ADE}$ or not, our results indicate that guanfacine might have the ability to prevent a slip from becoming a relapse after a period of prolonged abstinence.

It has previously been shown that a markedly higher guanfacine dose than used in the present study (1.5 vs $0.6 \mathrm{mg} / \mathrm{kg}$ ) decreased voluntary alcohol intake in alcoholpreferring AA rats (Opitz, 1990). However, in a pilot study, the dose of $1.5 \mathrm{mg} / \mathrm{kg}$ was clearly sedating (unpublished data from our research group), and $1 \mathrm{mg} / \mathrm{kg}$ of guanfacine induced sedative effects in the novel open field test (Smith and Aston-Jones, 2011). Thus, it is possible that the decreased alcohol intake previously observed by Opitz (1990) could be a nonspecific effect related to the sedative effects of guanfacine at a high dose. In this study, with the highest dose of $0.6 \mathrm{mg} / \mathrm{kg}$, the nonspecific effects are less likely as guanfacine significantly increased the water intake (in the presence of alcohol) and had no significant effect on water intake in alcohol-naive rats or voluntary intake of a salty solution. In addition, there was no significant effect on the inactive lever in the cue/priming-induced reinstatement experiment (although the highest guanfacine dose significantly decreased the number of inactive lever presses in the PR test (vehicle: $2.0 \pm 0.5$; guanfacine: $0.5 \pm 0.2$ lever presses). However, it should be noted that the general responding on the inactive lever was too low to be a reliable marker for possible locomotor deficits. Furthermore, previous studies showed that guanfacine $(0.5 \mathrm{mg} / \mathrm{kg}) \mathrm{did}$ not affect explorative behavior (Riga et al, 2014) or operant self-administration of sucrose (Le et al, 2011). Thus, collectively the present and previous results indicate that guanfacine's ability to attenuate voluntary alcohol intake, alcohol-seeking behavior, and cue/priming-induced reinstatement is most likely not caused by sedation or a general effect on locomotor behavior. 
Guanfacine's effect on human alcohol consumption remains to be investigated; however, we hypothesize that the present results showing that guanfacine attenuates several voluntary alcohol-mediated behaviors in animal models with predictive validity are suggestive of the putative effect of guanfacine as a novel AUD medication. The present and previous promising clinical and animal studies with guanfacine in different dependency disorders (Le et al, 2011; Fox et al, 2012, 2014; Riga et al, 2014), together with the findings that guanfacine appears to be safe and well tolerated in humans (Sallee et al, 2009a, b; Sallee and Eaton, 2010; Fox et al, 2012, 2014), merits evaluation of guanfacine's clinical efficacy in AUD patients.

\section{FUNDING AND DISCLOSURE}

The authors declare no conflict of interest.

\section{ACKNOWLEDGEMENTS}

We thank technicians Monica Aronsson, Linnea TörnerLarsson and Linnea Tankred, and undergraduate students Weinni Mussie and Jean Hussein for excellent assistance with the behavioral experiments. We thank Foundations Professor Rajita Sinha, Yale Medical University, for inspiring discussions regarding the potential of guanfacine in the treatment of dependence disorders. This study was funded by the Swedish Research Council (2009-2612), the Swedish Brain Foundation (FO2013-0042), and the Swedish Research Council for Health, Working Life and Welfare (2013-1781) to PS, and NordForsk (45782) to KJ.

\section{REFERENCES}

Anton RF, Oroszi G, O'Malley S, Couper D, Swift R, Pettinati H et al (2008). An evaluation of mu-opioid receptor (OPRM1) as a predictor of naltrexone response in the treatment of alcohol dependence: results from the Combined Pharmacotherapies and Behavioral Interventions for Alcohol Dependence (COMBINE) study. Arch Gen Psychiatry 65: 135-144.

Arnsten AF (2010). The use of $\alpha-2 \mathrm{~A}$ adrenergic agonists for the treatment of attention-deficit/hyperactivity disorder. Expert Rev Neurother 10: 1595-1605.

Arnsten AF (2011). Catecholamine influences on dorsolateral prefrontal cortical networks. Biol Psychiatry 69: e89-e99.

Arnsten AF, Jin LE (2014). Molecular influences on working memory circuits in dorsolateral prefrontal cortex. Prog Mol Biol Transl Sci 122: 211-231.

Arnsten AF, Steere JC, Hunt RD (1996). The contribution of alpha 2-noradrenergic mechanisms of prefrontal cortical cognitive function. Potential significance for attention-deficit hyperactivity disorder. Arch Gen Psychiatry 53: 448-455.

Arvanov VL, Liang X, Schwartz J, Grossman S, Wang RY (1997). Clozapine and haloperidol modulate N-methyl-D-aspartate- and non-N-methyl-D-aspartate receptor-mediated neurotransmission in rat prefrontal cortical neurons in vitro. J Pharmacol Exp Ther 283: 226-234.

Arvanov VL, Wang RY (1998). M100907, a selective 5-HT2A receptor antagonist and a potential antipsychotic drug, facilitates $\mathrm{N}$-methyl-D-aspartate-receptor mediated neurotransmission in the rat medial prefrontal cortical neurons in vitro. Neuropsychopharmacology 18: 197-209.

Avery RA, Franowicz JS, Studholme C, van Dyck CH, Arnsten AF (2000). The alpha-2A-adrenoceptor agonist, guanfacine, increases regional cerebral blood flow in dorsolateral prefrontal cortex of monkeys performing a spatial working memory task. Neuropsychopharmacology 23: 240-249.

Fernando AB, Economidou D, Theobald DE, Zou MF, Newman $\mathrm{AH}$, Spoelder $\mathrm{M}$ et al (2012). Modulation of high impulsivity and attentional performance in rats by selective direct and indirect dopaminergic and noradrenergic receptor agonists. Psychopharmacology (Berl) 219: 341-352.

Fox HC, Morgan PT, Sinha R (2014). Sex differences in guanfacine effects on drug craving and stress arousal in cocaine-dependent individuals. Neuropsychopharmacology 39: 1527-1537.

Fox HC, Seo D, Tuit K, Hansen J, Kimmerling A, Morgan PT et al (2012). Guanfacine effects on stress, drug craving and prefrontal activation in cocaine dependent individuals: preliminary findings. J Psychopharmacol 26: 958-972.

Gamo NJ, Arnsten AF (2011). Molecular modulation of prefrontal cortex: rational development of treatments for psychiatric disorders. Behav Neurosci 125: 282-296.

Goddard AW, Ball SG, Martinez J, Robinson MJ, Yang CR, Russell JM et al (2010). Current perspectives of the roles of the central norepinephrine system in anxiety and depression. Depress Anxiety 27: 339-350.

Goldstein RZ, Volkow ND (2011). Dysfunction of the prefrontal cortex in addiction: neuroimaging findings and clinical implications. Nat Rev Neurosci 12: 652-669.

Holmes A, Spanagel R, Krystal JH (2013). Glutamatergic targets for new alcohol medications. Psychopharmacology (Berl) 229: 539-554.

Konradsson A, Marcus MM, Hertel P, Svensson TH, Jardemark KE (2006). Inhibition of the glycine transporter GlyT-1 potentiates the effect of risperidone, but not clozapine, on glutamatergic transmission in the rat medial prefrontal cortex. Synapse 60: 102-108.

Le AD, Funk D, Juzytsch W, Coen K, Navarre BM, Cifani C et al (2011). Effect of prazosin and guanfacine on stress-induced reinstatement of alcohol and food seeking in rats. Psychopharmacology 218: 89-99.

Litten RZ, Ryan ML, Fertig JB, Falk DE, Johnson B, Dunn KE et al (2013). A double-blind, placebo-controlled trial assessing the efficacy of varenicline tartrate for alcohol dependence. J Addict Med 7: 277-286.

Marrs W, Kuperman J, Avedian T, Roth RH, Jentsch JD (2005). Alpha-2 adrenoceptor activation inhibits phencyclidine-induced deficits of spatial working memory in rats. Neuropsychopharmacology 30: 1500-1510.

McKee SA, Harrison EL, O’Malley SS, Krishnan-Sarin S, Shi J, Tetrault JM et al (2009). Varenicline reduces alcohol selfadministration in heavy-drinking smokers. Biol Psychiatry 66: 185-190.

McKinzie DL, Nowak KL, Yorger L, McBride WJ, Murphy JM, Lumeng $\mathrm{L}$ et al (1998). The alcohol deprivation effect in the alcohol-preferring $\mathrm{P}$ rat under free-drinking and operant access conditions. Alcohol Clin Exp Res 22: 1170-1176.

Mitchell JM, Teague CH, Kayser AS, Bartlett SE, Fields HL (2012). Varenicline decreases alcohol consumption in heavy-drinking smokers. Psychopharmacology 223: 299-306.

Opitz K (1990). The effect of clonidine and related substances on voluntary ethanol consumption in rats. Drug Alcohol Depend 25: 43-48.

Oslin DW, Berrettini W, Kranzler HR, Pettinati H, Gelernter J, Volpicelli JR et al (2003). A functional polymorphism of the $\mathrm{mu}$-opioid receptor gene is associated with naltrexone response in alcohol-dependent patients. Neuropsychopharmacology 28: $1546-1552$.

Rehm J, Mathers C, Popova S, Thavorncharoensap M, Teerawattananon Y, Patra J (2009). Global burden of disease and injury and economic cost attributable to alcohol use and alcohol-use disorders. Lancet 373: 2223-2233. 
Riga D, Schmitz LJ, van der Harst JE, van Mourik Y, Hoogendijk WJ, Smit AB et al (2014). A sustained depressive state promotes a guanfacine reversible susceptibility to alcohol seeking in rats. Neuropsychopharmacology 39: 1115-1124.

Sallee FR, Eaton K (2010). Guanfacine extended-release for attention-deficit/hyperactivity disorder (ADHD). Expert Opin Pharmacother 11: 2549-2556.

Sallee FR, Lyne A, Wigal T, McGough JJ (2009a). Long-term safety and efficacy of guanfacine extended release in children and adolescents with attention-deficit/hyperactivity disorder. J Child Adolesc Psychopharmacol 19: 215-226.

Sallee FR, McGough J, Wigal T, Donahue J, Lyne A, Biederman J (2009b). Guanfacine extended release in children and adolescents with attention-deficit/hyperactivity disorder: a placebocontrolled trial. J Am Acad Child Adolesc Psychiatry 48: 155-165.

Sanchis-Segura C, Spanagel R (2006). Behavioural assessment of drug reinforcement and addictive features in rodents: an overview. Addict Biol 11: 2-38.

Sica DA (2007). Centrally acting antihypertensive agents: an update. J Clin Hypertens (Greenwich) 9: 399-405.

Simms JA, Bito-Onon JJ, Chatterjee S, Bartlett SE (2010). LongEvans rats acquire operant self-administration of $20 \%$ ethanol without sucrose fading. Neuropsychopharmacology 35: 1453-1463.

Simms JA, Steensland P, Medina B, Abernathy KE, Chandler LJ, Wise $\mathrm{R}$ et al (2008). Intermittent access to $20 \%$ ethanol induces high ethanol consumption in Long-Evans and Wistar rats. Alcohol Clin Exp Res 32: 1816-1823.

Sinclair JD, Tiihonen K (1988). Lack of alcohol-deprivation effect in AA rats. Alcohol 5: 85-87.

Smith RJ, Aston-Jones G (2011). alpha(2) Adrenergic and imidazoline receptor agonists prevent cue-induced cocaine seeking. Biol Psychiatry 70: 712-719.

Spanagel R, Holter SM (2000). Pharmacological validation of a new animal model of alcoholism. J Neural Transm 107: 669-680.
Steensland P, Fredriksson I, Holst S, Feltmann K, Franck J, Schilstrom B et al (2012). The monoamine stabilizer (-)OSU6162 attenuates voluntary ethanol intake and ethanolinduced dopamine output in nucleus accumbens. Biol Psychiatry 72: 823-831.

Steensland P, Simms JA, Holgate J, Richards JK, Bartlett SE (2007). Varenicline, an \{alpha\}4beta2 nicotinic acetylcholine receptor partial agonist, selectively decreases ethanol consumption and seeking. Proc Natl Acad Sci USA 104: 12518-12523.

Steensland P, Simms JA, Nielsen CK, Holgate J, Bito-Onon JJ, Bartlett SE (2010). The neurokinin 1 receptor antagonist, ezlopitant, reduces appetitive responding for sucrose and ethanol. PLoS One 5: e12527.

Taylor FB, Russo J (2001). Comparing guanfacine and dextroamphetamine for the treatment of adult attention-deficit/hyperactivity disorder. J Clin Psychopharmacol 21: 223-228.

van Zwieten PA (1999). The renaissance of centrally acting antihypertensive drugs. J Hypertens Suppl 17: S15-S21.

Vengeliene V, Siegmund S, Singer MV, Sinclair JD, Li TK, Spanagel R (2003). A comparative study on alcohol-preferring rat lines: effects of deprivation and stress phases on voluntary alcohol intake. Alcohol Clin Exp Res 27: 1048-1054.

Wang M, Ramos BP, Paspalas CD, Shu Y, Simen A, Duque A et al (2007). Alpha2A-adrenoceptors strengthen working memory networks by inhibiting cAMP-HCN channel signaling in prefrontal cortex. Cell 129: 397-410.

Wise RA (1973). Voluntary ethanol intake in rats following exposure to ethanol on various schedules. Psychopharmacology (Berl) 29: 203-210.

This work is licensed under a Creative Commons Attribution-NonCommercial-NoDerivs 3.0 Unported License. To view a copy of this license, visit http:// creativecommons.org/licenses/by-nc-nd/3.0/

Supplementary Information accompanies the paper on the Neuropsychopharmacology website (http://www.nature.com/npp) 